\title{
A Retrospective Observational Study of Anlotinib in Patients with Platinum-Resistant or Platinum-Refractory Epithelial Ovarian Cancer
}

This article was published in the following Dove Press journal: Drug Design, Development and Therapy

\section{Qingli Cui \\ Yanhui Hu \\ Dongyang $\mathrm{Ma}$ \\ Huaimin Liu (D)}

Department of Integrated Traditional and Western Medicine, The Affiliated Cancer Hospital of Zhengzhou University, Zhengzhou City, Henan Province, People's Republic of China
Correspondence: Huaimin Liu Department of Integrated Traditional and Western Medicine, The Affiliated Cancer Hospital of Zhengzhou University, 127

Dongming Road, Zhengzhou 450008,

People's Republic of China

Email huaiminliu@sina.com
Objective: Anlotinib, an oral small-molecular tyrosine kinase inhibitor (TKI) on tumor angiogenesis and growth, has a wide spectrum of inhibitory effects on targets such as vascular endothelial growth factor receptors $2 / 3$ (VEGFR2/3), etc. The efficacy and safety of anlotinib in the treatment of platinum-resistant or platinum-refractory ovarian cancer were evaluated.

Patients and Methods: Patients with platinum-resistant or platinum-refractory ovarian cancer that treated with anlotinib in the Affiliated Cancer Hospital of Zhengzhou University from May 2018 to March 2020 were included. Medical records were reviewed in terms of objective response, survival outcomes, and safety.

Results: A total of 38 patients were analyzed. The median progression-free survival and the median overall survival were 7.7 months (95\% CI: 6.7-8.7) and 16.5 months (95\% CI: 13.3-19.7), respectively. About 17 patients received anlotinib monotherapy, and the median progression-free survival was 7.7 months (95\% CI: 6.3-9.1). A total of 19 cases received anlotinib plus chemotherapy with a median progression-free survival of 8.0 months $(95 \% \mathrm{CI}$ : 4.8-11.2). A total of 2 cases received anlotinib plus anti-PD-1 antibody pembrolizumab, and 1 case had partial response, the other progressive disease. The objective response rate was $42.1 \%$ while the disease control rate was $86.8 \%$. A total of 5 patients experienced dose reduction from $12 \mathrm{mg}$ to $10 \mathrm{mg}$ because of adverse effects. The most common adverse effects were hypertension (31.6\%), fatigue (28.9\%), anorexia (26.3\%) and hand-foot syndrome $(23.7 \%)$. No treatment-related deaths were recorded.

Conclusion: Anlotinib produced moderate improvements in progression-free survival and overall survival in patients with platinum-resistant or platinum-refractory ovarian cancer. It indicates that anlotinib maybe a new treatment option for patients with platinum-resistant or platinum-refractory ovarian cancer.

Keywords: angiogenesis, anlotinib, ovarian cancer, platinum-resistant

\section{Introduction}

According to Global Cancer Statistics 2018, ovarian cancer is the second most common cause of death related to gynecologic malignancy and the eighth leading cause of cancer-related death in women worldwide. ${ }^{1}$ More than $70 \%$ of patients are diagnosed as advanced stage. ${ }^{2}$ Despite the majority of patients achieve clinical complete remission after chemotherapy, over $50 \%$ of patients with advanced ovarian cancer will relapse and cause death due to the emerging resistance to chemotherapy, particularly resistance to platinum. ${ }^{3}$ The treatment of patients with platinum-resistant or platinum-refractory disease remains a challenge. ${ }^{4}$ 
Angiogenesis plays a pivotal role in normal ovarian physiology as well as in the progression of ovarian cancer through ascites formation and metastatic spread. Bevacizumab, the first active targeted agent in ovarian cancer, which targets angiogenesis by binding to VEGFA, effectively blocking the interaction of VEGF and its receptor. ${ }^{5}$ The AURELIA trial showed that adding bevacizumab to chemotherapy in patients with platinumresistant disease significantly improved the PFS (3.4 vs 6.7 months; hazard ratio (HR), $0.48 ; \mathrm{P}=0.001$ ) and ORR (11.8\% vs $27.3 \% ; \mathrm{P}=0.001)$, but the OS trend (13.3 vs 16.6 months; HR, 0.85; $\mathrm{P}=0.171)$ was not significant. ${ }^{6}$ Moreover, increasing evidence has suggested that multireceptor tyrosine kinase inhibitors (TKIs; including VEGF receptor inhibitors) such as nintedanib, pazopanib, apatinib, improve the outcome of platinum-resistant ovarian cancer patients. ${ }^{7-10}$ Some studies have shown that the combination of chemotherapy and the angiogenesis inhibitor was effective for platinum-resistant or platinumrefractory ovarian cancer. ${ }^{11,12}$ Some researchers have explored the combination of anti-angiogenic therapy and immunotherapy in ovarian cancer. ${ }^{13}$

Anlotinib is an oral small-molecular TKI with the suppression of tumor growth and angiogenesis. ${ }^{14}$ It has a wide spectrum of inhibitory effects on targets such as vascular endothelial growth factor receptors $2 / 3$ (VEGFR2/3), fibroblast growth factor receptor 1-4 (FGFR1-4), platelet-derived growth factor receptors $a / b$ (PDGFR a/b), c-kit, and Ret. ${ }^{15,16}$ It has been reported that anlotinib combined with etoposide has anti-tumor effects on platinum-resistant ovarian cancer patients. ${ }^{17}$ Hereon, this study was to evaluate the efficacy and safety of anlotinib in patients with platinum-resistant or platinum-refractory ovarian cancer in our center.

\section{Patients and Methods}

\section{Study Population}

The present study was approved by the ethics committee of The Affiliated Cancer Hospital of Zhengzhou University (201910226). Clinical information and follow-up data were collected from patients with platinum-resistant (defined as progression within 6 months after the last platinum treatment) or platinum-refractory (defined as progression during the initial platinum-based treatment) ovarian cancer who received anlotinib between May 2018 to March 2020 in our hospital. All patients took anlotinib for more than 2 cycles, regardless of combination therapy or monotherapy.

\section{Treatment}

Anlotinib was administered orally at $10 \mathrm{mg}$ or $12 \mathrm{mg}$ daily on days 1-14. 21 days was defined as one cycle. 2 patients took anlotinib combined with anti-PD-1 antibody pembrolizumab, 19 patients took anlotinib combined with chemotherapy, and 17 patients treated with anlotinib monotherapy. The dosage of anlotinib was determined by the attending physician based on the patients' age, body weight, general condition and tolerance, and comprehensive treatment.

\section{Efficacy and Safety}

Survival and anlotinib discontinuation in all patients were monitored until July 1 2020. They were followed up with CA125 or imaging examination. Tumor response was assessed by investigators using $\mathrm{CT}$ scans or MRI. Treatment responses were evaluated through the Response Evaluation Criteria for Solid Tumors (RECIST version $1.1^{18}$ ), including complete response (CR), partial response (PR), stable disease (SD), and progressive disease (PD). Objective response rate (ORR) was measured as the proportion of eligible patients who achieved confirmed complete response or partial response. Disease control rate (DCR) was referred to the proportion of patients who achieved complete response, partial response, and stable disease for at least 8 weeks. Progression-free survival (PFS) was defined as the time from initiation of anlotinib until disease progression or death. During the last follow-up, patients without disease progression were considered censored. The overall survival (OS) referred to the time from initiation of anlotinib administration to death for any cause or the last follow-up visit for living patients. Safety was monitored by recording patients, chief complaint, physical examinations, vital signs, adverse events, as well as hematology and clinical chemistry tests. Adverse events were graded according to National Cancer Institute Common Terminology Criteria for Adverse Events (NCI CTCAE) version 5.0.

\section{Statistical Analysis}

Descriptive statistics were used to describe patients' clinical and demographic characteristics. The Kaplan-Meier method was used to estimate PFS and OS. All statistical analyses were done by IBM SPSS ver. 23.0 (IBM Corp., Armonk, NY, USA). 


\section{Results}

\section{Patient Characteristics}

A total of 38 patients were included in this study. The baseline characteristics of the patients were summarized in Table 1. The median age at diagnosis of recurrence was 56 years (range,44-84 years). 31 patients had serous adenocarcinoma, 3 cases had endometrioid carcinoma, and 4 cases had mixed serous and endometrioid carcinoma. There are 33 platinum-resistant patients and 5 platinumrefractory patients. All patients were diagnosed with advanced-stage disease (stage III: $n=15$; stage IV: $n=23$ ). The median number of previous regimens was 4 (range $=$ 2-11), in particular, $92.1 \%$ of patients had received $\geq 3$ previous line, and $31.6 \%$ of patients had received $\geq 5$ line treatments before anlotinib was administered. Before anlotinib, 7 patients had received anti-angiogenic therapy, including 4 with bevacizumab and 3 with apatinib. 6 patients had been treated with PARP (poly ADP-ribose polymerase) inhibitors, 5 patients had received olaparib, and 1 patient had received niraparib. In this study, 17 patients received anlotinib monotherapy, 19 patients received anlotinib combined with chemotherapy, and 2 patients received anlotinib combined with anti-PD-1 antibody pembrolizumab.

\section{Efficacy}

Median follow-up time at the time of data analysis (July 1, 2020) was 10.25 months (range $=3.0-23.5$ ). At the time of data cut-off, 27 of the 38 patients had discontinued anlotinib and 11 patients were continuing anlotinib treatment. The median PFS was 7.7 months (95\% CI: 6.7-8.7) and the OS was 16.5 months (95\% CI: 13.3-19.7) (Figure 1). A total of 5 patients progressed and 16 patients had a partial response, none had the complete response. Besides, 17 patients were in stable condition. Thus, the ORR and DCR were $42.1 \%$ and $86.8 \%$, respectively.

\section{Anlotinib Monotherapy}

17 cases received anlotinib monotherapy (Table 2). The median progression-free survival was 7.7 months $(95 \% \mathrm{CI}$ : 6.3-9.1) and the median overall survival was not reached. It is worth mentioning that among these 17 patients, 4 cases had partial response, and have not yet reached progress with a PFS beyond 11 months. 10 patients were in stable condition $(58.5 \%)$ and 3 patients suffered from
Table I Baseline Characteristics in 38 Patients. Values are Reported as Frequency ( $\mathrm{n}[\%]$ ) or as Mean (Range)

\begin{tabular}{|c|c|}
\hline Characteristic of Patients (Percent) & $\mathbf{N}=38(\%)$ \\
\hline \multicolumn{2}{|l|}{ Age, years } \\
\hline Median, IQR & $56(5 I-64.25)$ \\
\hline \multicolumn{2}{|l|}{ ECOG PS, n (\%) } \\
\hline 0 & $\mathrm{I}(2.6 \%)$ \\
\hline I & $25(65.8 \%)$ \\
\hline 2 & $12(31.6 \%)$ \\
\hline \multicolumn{2}{|l|}{ Histology, n (\%) } \\
\hline Serous & $31(81.6 \%)$ \\
\hline Endometrioid & $3(7.9 \%)$ \\
\hline Mixed serous and endometrioid & $4(10.5 \%)$ \\
\hline \multicolumn{2}{|l|}{ BRCA status, $n$ (\%) } \\
\hline BRCA I/2 mutation & $5(\mid 3.2 \%)$ \\
\hline Wild type & $17(44.7 \%)$ \\
\hline Unknown & $16(42.1 \%)$ \\
\hline \multicolumn{2}{|l|}{ International FIGO stage } \\
\hline III & $15(39.5 \%)$ \\
\hline IV & $23(60.5 \%)$ \\
\hline \multicolumn{2}{|c|}{ Previous lines of chemotherapy or target therapy } \\
\hline $1-2$ & $4(10.5 \%)$ \\
\hline $3-5$ & $26(68.4 \%)$ \\
\hline$\geq 6$ & $8(21.1 \%)$ \\
\hline \multicolumn{2}{|l|}{ Prior anti-angiogenic therapy } \\
\hline Yes & $7(18.4 \%)$ \\
\hline No & $31(81.6 \%)$ \\
\hline \multicolumn{2}{|l|}{ Prior PARP inhibitor } \\
\hline Yes & $6(15.8 \%)$ \\
\hline No & $32(84.2 \%)$ \\
\hline \multicolumn{2}{|l|}{ Dose reduction } \\
\hline Yes & $5(\mid 3.2 \%)$ \\
\hline No & $33(86.8 \%)$ \\
\hline \multicolumn{2}{|l|}{ Treatment status } \\
\hline Monotherapy & $17(44.7 \%)$ \\
\hline Combined with chemotherapy & $19(50 \%)$ \\
\hline Combined with pembrolizumab & $2(5.3 \%)$ \\
\hline \multicolumn{2}{|l|}{ CAI 25 before anlotinib administration } \\
\hline$\leq 35 \mathrm{Ul} / \mathrm{mL}$ & $4(10.5 \%)$ \\
\hline$>35 \mathrm{Ul} / \mathrm{mL}$ & $34(89.5 \%)$ \\
\hline
\end{tabular}



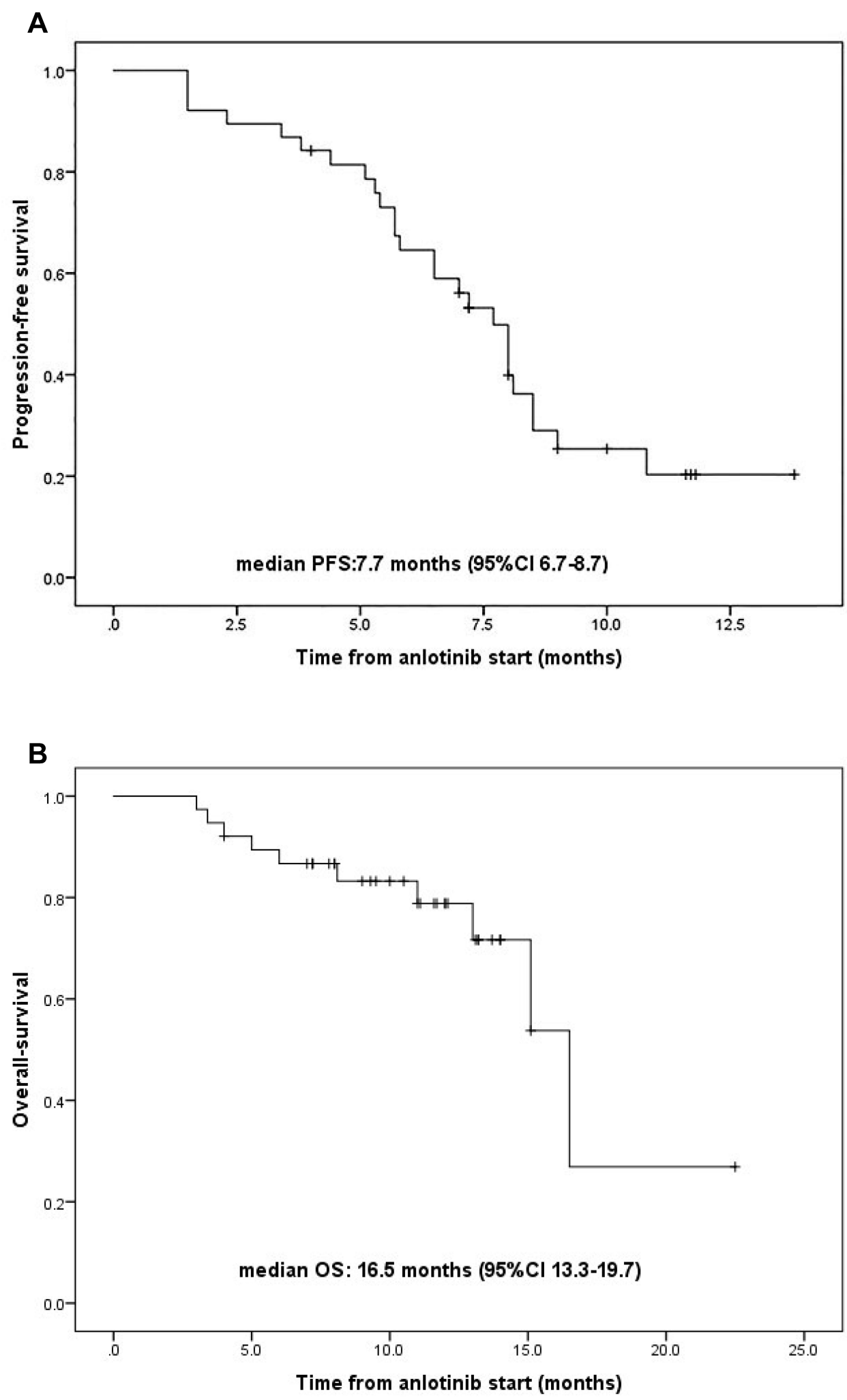

Figure I Kaplan-Meier estimates of PFS (A) and OS (B) of 38 patients.

Abbreviations: PFS, progression-free survival; OS, Overall survival. 
Table 2 Short-Term Efficacy of Anlotinib Monotherapy

\begin{tabular}{|l|l|}
\hline Short-Term Efficacy & Number of Patients (Percent) \\
\hline Complete response (CR) & $0(0 \%)$ \\
Partial response (PR) & $4(23.5 \%)$ \\
Stable disease (SD) & $10(58.8 \%)$ \\
Progressive disease (PD) & $3(17.6 \%)$ \\
\hline
\end{tabular}

Note: Short-term efficacy was classified by modified Response Evaluation Criteria in Solid Tumors version I.I (RECIST I.I).

disease progression. (17.6\%). Therefore, the ORR was $23.5 \%$, and the DCR was $82.3 \%$.

\section{Anlotinib Combined with Chemotherapy}

A total of 19 cases were administrated anlotinib plus chemotherapy, of which 15 patients combined with etoposide capsules, 1 patient combined with capecitabine, 1 patient combined with epirubicin, 1 patient combined with docetaxel, and 1 patient combined with lobaplatin. The median progression-free survival was 8.0 months (95\% CI: 4.8-11.2) and the median overall survival was not reached. 7 patients had a partial response and 11 patients in stable condition. The ORR and DCR of patients treated with anlotinib combined with chemotherapy were $36.8 \%$ and $94.7 \%$, respectively (Table 3 ).

\section{Anlotinib Combined with Anti-PD-I Antibody Pembrolizumab}

Two patients received anlotinib combined with pembrolizumab. 1 patient progressed after 2 cycles administration of anlotinib plus anti-PD-1 antibody pembrolizumab. 1 patient achieved partial response and lasted over 7.2 months (Table 4).

\section{Dose Adjustment and Safety}

There are 5 patients (13.2\%) started from the daily dose of $10 \mathrm{mg}$ and none had a dose reduction. Other 33 patients $(86.8 \%)$ started from $12 \mathrm{mg}$ daily, in which 5 patients $(15.2 \%)$ required dose reduction to $10 \mathrm{mg}$ after the evaluation of adverse events. 3 patients (7.9\%) developed severe hand-foot syndrome, 1 patient $(2.6 \%)$ had intolerable sore

Table 3 Short-Term Efficacy of Anlotinib Combined with Chemotherapy

\begin{tabular}{|l|l|}
\hline Short-Term Efficacy & Number of Patients (Percent) \\
\hline Complete response (CR) & $0(0 \%)$ \\
Partial response (PR) & $7(36.8 \%)$ \\
Stable disease (SD) & $\mathrm{Il}(57.9 \%)$ \\
Progressive disease (PD) & $\mathrm{I}(5.3 \%)$ \\
\hline
\end{tabular}

Table 4 Short-Term Efficacy of Anlotinib Combined with AnitPD-I Antibody

\begin{tabular}{|l|l|}
\hline Short-Term Efficacy & Number of Patients (Percent) \\
\hline Complete response (CR) & $0(0 \%)$ \\
Partial response (PR) & $\mathrm{I}(50 \%)$ \\
Stable disease (SD) & $0(0 \%)$ \\
Progressive disease (PD) & $\mathrm{I}(50 \%)$ \\
\hline
\end{tabular}

throat, and 1 patient $(2.6 \%)$ developed severe proteinuria. The most common adverse events were hypertension (31.6\%), fatigue $(28.9 \%)$, anorexia (26.3\%), hand and foot syndrome (23.7\%), mainly as grade 1 to 2 (Table 5).

\section{Discussion}

Although ovarian cancer is usually a chemosensitive disease, patients with advanced stages inevitably relapse, eventually leading to chemotherapy resistance, especially platinum resistance. For platinum-resistant disease, NCCN guidelines version 1.2020 (ovarian/fallopian tube/primary peritoneal cancer) recommend clinical trials, optimal supportive care, and relapse treatment, with consideration of a highly individualistic basis. Non-platinum-based agents or regimens are preferred (ie, docetaxel, oral etoposide, gemcitabine, weekly paclitaxel with or without pazopanib, liposomal doxorubicin with or without bevacizumab, weekly paclitaxel/bevacizumab) sequential therapy is usually performed with a single agent. ${ }^{6,11,19-22}$ Although notable progress has been made in the treatment of ovarian cancer, the prognosis of patients with the resistant or refractory disease remains poor. ${ }^{4,23}$

Beyond chemotherapy, molecular targeted therapy plays an important role in the treatment of platinumresistant or platinum-refractory ovarian cancer. Olaparib is a PARP inhibitor that has a higher response rate in patients with BRCA1 or BRCA2 mutations than those with BRCA negative mutations, especially in platinumsensitive diseases. ${ }^{24}$ If the disease is resistant or refractory to platinum, then a lower response rate to olaparib is observed. $^{25-27}$ Therefore, the effect of PARP inhibitor on platinum-resistant disease is limited.

Anti-angiogenesis is considered as a promising target for the treatment of platinum-resistant ovarian cancer. ${ }^{5}$ Bevacizumab has single-agent activity in patients with platinum-resistant ovarian cancer or peritoneal serous cancer with a median PFS of 4.4 months ( $95 \%$ CI, 3.1 to 5.5 months). ${ }^{28}$ Bevacizumab combined with chemotherapy shown improved activity in platinum-resistant ovarian cancer. ${ }^{6,11,12,29-32}$ In AURELIA, the PFS of chemotherapy 
Table 5 Treatment-Related Toxicities

\begin{tabular}{|c|c|c|c|c|c|}
\hline & Grade I & Grade 2 & Grade 3 & Grade 4 & Total $n(\%)$ \\
\hline Hypertension & 8 & 3 & 1 & 0 & 31.6 \\
\hline Fatigue & 8 & 2 & 1 & 0 & 28.9 \\
\hline Anorexia & 6 & 4 & 0 & 0 & 26.3 \\
\hline Hand-foot syndrome & 5 & 2 & 3 & 0 & 23.7 \\
\hline Diarrhea & 5 & 3 & 0 & 0 & 21.1 \\
\hline Proteinuria & 3 & 2 & 1 & 0 & 21.1 \\
\hline Sore throat & 2 & I & 1 & 0 & 13.2 \\
\hline Dyspepsia & 3 & 2 & 0 & 0 & 13.2 \\
\hline Dry mouth & 7 & 2 & 0 & 0 & 23.7 \\
\hline Weight loss & 5 & 3 & 0 & 0 & 21.1 \\
\hline Nausea & 4 & 2 & 0 & 0 & 15.8 \\
\hline Hypoproteinemia & 3 & 2 & 1 & 0 & 15.8 \\
\hline Vomiting & 2 & 1 & 0 & 0 & 7.9 \\
\hline Mucositis oral & 3 & 1 & 0 & 0 & 10.5 \\
\hline Headache & 2 & I & 0 & 0 & 7.9 \\
\hline Hoarseness & 3 & 2 & 0 & 0 & 13.2 \\
\hline Pharyngodynia & 3 & 1 & 0 & 0 & 10.5 \\
\hline Neutropenia & 5 & 2 & 1 & 0 & 21.1 \\
\hline Thrombocytopenia & 7 & 1 & 1 & 0 & 23.7 \\
\hline Anaemia & 5 & 3 & 0 & 0 & 21.1 \\
\hline
\end{tabular}

plus bevacizumab in patients with platinum-resistant disease is 6.7 months. However, the prolongation of OS in the bevacizumab group did not reach a significant level. ${ }^{6}$ AntiVEGF tyrosine kinase inhibitors, pazopanib or cediranib, have also been tested with weekly paclitaxel in refractory or resistant ovarian cancer. The results showed that cediranib had some certain effect, but it is not available in mainland China, and pazopanib was limited by its toxicity. ${ }^{19,33,34}$ Apatinib, an oral tyrosine kinase inhibitor against VEGFR-2, has shown activity in recurrent ovarian cancer patients resistant to platinum. ${ }^{10,35}$ The median PFS of patients treated with apatinib plus etoposide capsule is 8.1 months and dose reduction occurred in $82 \%$ patients for apatinib. In a retrospective study, ${ }^{36}$ apatinib as maintenance therapy following chemotherapy had a median PFS of 6.0 months and a median OS of 11.0 months. The patients taking apatinib as salvage therapy had a DCR of $81.8 \%$ and a median PFS of 3.0 months. The most common adverse effects were hand-foot syndrome $(53.57 \%)$, secondary hypertension $(46.43 \%)$, and fatigue (14.29\%). Thus, the adverse reactions of apatinib also limit its widespread use.

Anlotinib is a new orally administered tyrosine kinase inhibitor. The preclinical studies have suggested that anlotinib has a better anti-angiogenic effect than sunitinib, sorafenib, and nintedanib. ${ }^{16}$ The latter are the three main anti-angiogenic drugs at present and have anti-tumor activity against advanced ovarian cancer. ${ }^{23,37,38}$ In terms of grade 3 or higher, anlotinib has a lower incidence of toxic effects than sunitinib. ${ }^{39}$ Therefore, anlotinib is a potential agent to inhibit angiogenesis and be safely applied to ovarian cancer. Our results showed that in 38 women with platinum-resistant or platinum-refractory ovarian cancer, the median progression-free survival of anlotinib was 7.7 months (95\% CI: 6.7-8.7), and the overall survival time was 16.5 months (95\% CI: 13.3-19.7). In 17 patients who received anlotinib monotherapy, the DCR was $82.3 \%$, the median PFS was 7.7 months $(95 \% \mathrm{CI}$ : 6.3-9.1), and the median overall survival was not achieved. The most common adverse reaction was hypertension (31.6\%), fatigue (28.9\%), anorexia (26.3\%), hand and foot syndrome $(23.7 \%)$, mainly as grade 1 to 2 . In this study, anlotinib alone achieved PFS of 7.7 months, which was not inferior to bevacizumab of 4.4 months. ${ }^{28}$ This might be due to the fact that anlotinib has multiple antiangiogenic targets. Compared with apatinib or pazopanib, it had lower toxicity, and patients were more likely to be exposed to the drug for a long time.

Novel multi-target tyrosine kinase inhibitor plus a single chemotherapy regimen was considered as 
a valuable treatment strategy for platinum-resistant ovarian cancer. This study reported the clinical outcomes for 19 patients with platinum-resistant ovarian cancer who received anlotinib combined with chemotherapy. A median PFS of 8 months (95\% CI: 4.8-11.2) was observed. Compared with anlotinib monotherapy, the benefits are not significant. We believe it might be related to the treatment history of this group of patients. This needs further study.

Immunotherapy shows good prospects in other types of tumors, such as lung cancer, but the effect of immunotherapy is not satisfactory for ovarian cancer, especially platinum-resistant ovarian cancer. In KEYNOTE-028 and KEYNOTE-100, the effect of pembrolizumab in ovarian cancer is modest, with ORRs ranging from 8 to $22 \%$. ${ }^{40,41}$ In KEYNOTE-100 study, although patients with higher tumor PD-L1 expression had higher ORR, the predictive value of PD-L1 in ovarian cancer is not yet well clear. ${ }^{41}$ The angiogenesis is beneficial to tumor growth and spread in the tumor microenvironment (TME), which has been well established. $^{42}$ Within the TME, tumor angiogenesis and immune suppression play interconnected roles in promoting tumor progression and metastasis. ${ }^{43}$ The cytokines and angiogenic factors released by TME cells, such as VEGF, TGF $\beta$, and prostaglandin E2 (PGE2), mediate immunosuppression through reducing antigen presentation to $\mathrm{T}$ cells and the effector response of T cells. ${ }^{44}$ Preclinical data strongly suggest that antiangiogenic treatment facilitates the arrival of immune effectors and reduces the presence of myeloid cells involved in immune suppression, which could translate into a possible synergistic effect with immunomodulators. ${ }^{45}$ Here, two cases were administrated anlotinib combined with anti-PD-1 antibody pembrolizumab, 1 patient suffered disease progression after 2 cycles of treatment, and 1 patient is still alive with no evidence of disease progression over 7.2 months. This suggests that the combination of antiangiogenesis and immunotherapy may be a promising therapy for ovarian cancer. However, there are just two cases, and the efficacy of anlotinib combined with the anti-PD-1 antibody on ovarian cancer still needs further verification.

This is a retrospective study to evaluate the side effects and short-term efficacy of anlotinib in platinum-resistant or platinum-refractory ovarian cancer patients. All patients in this study have been heavily treated and considered as platinum-resistant or refractory, but have achieved good clinical benefits. It is acknowledged that this study had some limitations such as not a randomized controlled study, small sample size, short observation time, etc. We believe that this study can provide a reference for the research of anlotinib in ovarian cancer.

In conclusion, anlotinib might be a promising agent for platinum-resistant or platinum-refractory ovarian cancer with lower toxicity. Further investigations are warranted to verify the efficacy of anlotinib in the treatment of patients with ovarian cancer.

\section{Ethics Approval and Consent to Participate}

This study was approved by the ethics committee of The Affiliated Cancer Hospital of Zhengzhou Univesity (201910226). All patients' consent was informed consent and this study was conducted in accordance with the Declaration of Helsinki.

\section{Acknowledgments}

We sincerely appreciate Xueli Jing, Shanshan Zhan, Baoliang Chen, Zezhong Li, Jia Li, Yanyan Qi, Xiang Ma for their help in the preliminary work.

\section{Funding}

There were no sources of funding or financial support for this manuscript.

\section{Disclosure}

The authors report no conflicts of interest in this work.

\section{References}

1. Bray F, Ferlay J, Soerjomataram I, et al. Global cancer statistics 2018: GLOBOCAN estimates of incidence and mortality worldwide for 36 cancers in 185 countries[J]. CA Cancer J Clin. 2018;68(6):394-424. doi:10.3322/caac. 21492

2. Siegel RL, Miller KD, Jemal A. Cancer statistics, 2018[J]. CA Cancer J Clin. 2018;68(1):7-30.

3. Ozols RF. Systemic therapy for ovarian cancer: current status and new treatments[J]. Semin Oncol. 2006;33(2 Suppl 6):S3-S11.

4. Pujade-Lauraine E, Combe P. Recurrent ovarian cancer[J]. Ann Oncol. 2016;27(Suppl 1):i63-i65.

5. Monk BJ, Minion LE, Coleman RL. Anti-angiogenic agents in ovarian cancer: past, present, and future[J]. Ann Oncol. 2016;27(Suppl 1):i33i39.

6. Pujade-Lauraine E, Hilpert F, Weber B, et al. Bevacizumab combined with chemotherapy for platinum-resistant recurrent ovarian cancer: the AURELIA open-label randomized Phase III trial[J]. J Clin Oncol. 2014;32(13):1302-1308.

7. Ledermann JA, Hackshaw A, Kaye S, et al. Randomized Phase II placebo-controlled trial of maintenance therapy using the oral triple angiokinase inhibitor BIBF 1120 after chemotherapy for relapsed ovarian cancer[J]. J Clin Oncol. 2011;29(28):3798-3804.

8. Bois A, Kristensen G, Ray-Coquard I, et al. Standard first-line chemotherapy with or without nintedanib for advanced ovarian cancer (AGO-OVAR 12): a randomised, double-blind, placebo-controlled Phase 3 trial[J]. Lancet Oncol. 2016;17(1):78-89. 
9. J W K, Mahner S, L Y W, et al. Pazopanib Maintenance Therapy in East Asian Women With Advanced Epithelial Ovarian Cancer: results From AGO-OVAR16 and an East Asian Study[J]. Int $J$ Gynecol Cancer. 2018;28(1):2-10.

10. C Y L, Wang Y, Xiong Y, et al. Apatinib combined with oral etoposide in patients with platinum-resistant or platinum-refractory ovarian cancer (AEROC): a Phase 2, single-arm, prospective study[J]. Lancet Oncol. 2018;19(9):1239-1246.

11. Nagao S, Kogiku A, Suzuki K, et al. A phase II study of the combination chemotherapy of bevacizumab and gemcitabine in women with platinum-resistant recurrent epithelial ovarian, primary peritoneal, or fallopian tube cancer[J]. J Ovarian Res. 2020;13(1):14.

12. Mcgonigle KF, Muntz HG, Vuky J, et al. Combined weekly topotecan and biweekly bevacizumab in women with platinum-resistant ovarian, peritoneal, or fallopian tube cancer: results of a phase 2 study[J]. Cancer. 2011;117(16):3731-3740.

13. Garcia-Martinez E, Redondo A, Piulats JM, et al. Are antiangiogenics a good 'partner' for immunotherapy in ovarian cancer?[J]. Angiogenesis. 2020.

14. Syed YY. Anlotinib: first Global Approval[J]. Drugs. 2018;78 (10):1057-1062.

15. Yang Q, Ni L, Imani S, et al. Anlotinib Suppresses Colorectal Cancer Proliferation and Angiogenesis via Inhibition of AKT/ERK Signaling Cascade[J]. Cancer Manag Res. 2020;12:4937-4948.

16. Lin B, Song X, Yang D, et al. Anlotinib inhibits angiogenesis via suppressing the activation of VEGFR2, PDGFRbeta and FGFR1[J]. Gene. 2018;654:77-86.

17. Sun L, Yang M, Zhang X, et al. Anlotinib combined with etoposide for platinum-resistant recurrent ovarian cancer: A case report[J]. Medicine. 2020;99(20):e20053.

18. Eisenhauer EA, Therasse P, Bogaerts J, et al. New response evaluation criteria in solid tumours: revised RECIST guideline (version 1.1) [J]. Eur J Cancer. 2009;45(2):228-247.

19. Pignata S, Lorusso D, Scambia G, et al. Pazopanib plus weekly paclitaxel versus weekly paclitaxel alone for platinum-resistant or platinum-refractory advanced ovarian cancer (MITO 11): a randomised, open-label, phase 2 trial[J]. Lancet Oncol. 2015;16 (5):561-568.

20. Elit L, Hirte H. Palliative systemic therapy for women with recurrent epithelial ovarian cancer: current options[J]. Onco Targets Ther. 2013;6:107-118.

21. Shoji T, Komiyama S, Kigawa J, et al. An open-label, randomized, phase II trial evaluating the efficacy and safety of standard of care with or without bevacizumab in platinum-resistant epithelial ovarian, fallopian tube, or primary peritoneal cancer patients previously treated with bevacizumab for front-line or platinum-sensitive ovarian cancer: rationale, design, and methods of the Japanese Gynecologic Oncology Group study JGOG3023[J]. BMC Cancer. 2018;18(1):771.

22. Chekerov R, Hilpert F, Mahner S, et al. Sorafenib plus topotecan versus placebo plus topotecan for platinum-resistant ovarian cancer (TRIAS): a multicentre, randomised, double-blind, placebo-controlled, phase 2 trial[J]. Lancet Oncol. 2018;19 (9): 1247-1258.

23. Griffiths RW, Y K Z, Evans S, et al. Outcomes after multiple lines of chemotherapy for platinum-resistant epithelial cancers of the ovary, peritoneum, and fallopian tube[J]. Int J Gynecol Cancer. 2011;21 (1):58-65.

24. Ledermann J, Harter P, Gourley C, et al. Olaparib maintenance therapy in patients with platinum-sensitive relapsed serous ovarian cancer: a preplanned retrospective analysis of outcomes by BRCA status in a randomised phase 2 trial[J]. Lancet Oncol. 2014;15 (8):852-861.

25. Matulonis UA, Penson RT, Domchek SM, et al. Olaparib monotherapy in patients with advanced relapsed ovarian cancer and a germline BRCA1/2 mutation: a multistudy analysis of response rates and safety[J]. Ann Oncol. 2016;27(6):1013-1019.
26. Audeh MW, Carmichael J, R T P, et al. Oral poly(ADP-ribose) polymerase inhibitor olaparib in patients with BRCA1 or BRCA2 mutations and recurrent ovarian cancer: a proof-of-concept trial[J]. Lancet. 2010;376(9737):245-251.

27. Fong PC, Yap TA, Boss DS, et al. Poly(ADP)-ribose polymerase inhibition: frequent durable responses in BRCA carrier ovarian cancer correlating with platinum-free interval[J]. J Clin Oncol. 2010;28 (15):2512-2519.

28. Cannistra SA, Matulonis UA, Penson RT, et al. Phase II study of bevacizumab in patients with platinum-resistant ovarian cancer or peritoneal serous cancer[J]. J Clin Oncol. 2007;25(33):5180-5186.

29. Niu J, Kundranda MN, Markman M, et al. Platinum-GemcitabineAvastin (PGA) for platinum-resistant/refractory ovarian cancer[J]. Eur J Gynaecol Oncol. 2017;38(1):40-44.

30. Verschraegen CF, Czok S, C Y M, et al. Phase II study of bevacizumab with liposomal doxorubicin for patients with platinum- and taxane-resistant ovarian cancer[J]. Ann Oncol. 2012;23 (12):3104-3110.

31. Tillmanns TD, Lowe MP, Walker MS, et al. Phase II clinical trial of bevacizumab with albumin-bound paclitaxel in patients with recurrent, platinum-resistant primary epithelial ovarian or primary peritoneal carcinoma[J]. Gynecol Oncol. 2013;128(2):221-228.

32. Poveda AM, Selle F, Hilpert F, et al. Bevacizumab combined with weekly paclitaxel, pegylated liposomal doxorubicin, or topotecan in platinum-resistant recurrent ovarian cancer: analysis by chemotherapy cohort of the randomized phase iii AURELIA trial[J]. $J$ Clin Oncol. 2015;33(32):3836-3838.

33. Richardson DL, M W S, R L C, et al. Paclitaxel With and Without Pazopanib for Persistent or Recurrent Ovarian Cancer: A Randomized Clinical Trial[J]. JAMA Oncol. 2018;4(2):196-202.

34. Hirte H, Lheureux S, Fleming GF, et al. A phase 2 study of cediranib in recurrent or persistent ovarian, peritoneal or fallopian tube cancer: a trial of the Princess Margaret, Chicago and California Phase II Consortia[J]. Gynecol Oncol. 2015;138(1):55-61.

35. Miao M, Deng G, Luo S, et al. A phase II study of apatinib in patients with recurrent epithelial ovarian cancer[J]. Gynecol Oncol. 2018;148(2):286-290.

36. Zhang J, Li A, Jiang Q, et al. Efficacy And Safety Of Apatinib Treatment In Platinum-Resistant Recurrent Epithelial Ovarian Cancer: A Real World Study[J]. Drug Des Devel Ther. 2019;13:3913-3918.

37. Du Bois A, Kristensen G, Ray-Coquard I, et al. Standard first-line chemotherapy with or without nintedanib for advanced ovarian cancer (AGO-OVAR 12): a randomised, double-blind, placebo-controlled phase 3 trial[J]. Lancet Oncol. 2016;17(1):78-89.

38. Baumann KH, Du Bois A, Meier W, et al. A phase II trial (AGO 2.11) in platinum-resistant ovarian cancer: a randomized multicenter trial with sunitinib (SU11248) to evaluate dosage, schedule, tolerability, toxicity and effectiveness of a multitargeted receptor tyrosine kinase inhibitor monotherapy[J]. Ann Oncol. 2012;23(9):2265-2271.

39. Zhou AP, Bai Y, Song Y, et al. Anlotinib Versus Sunitinib as First-Line Treatment for Metastatic Renal Cell Carcinoma: A Randomized Phase II Clinical Trial[J]. Oncologist. 2019;24(8): e702-e708.

40. Varga A, Piha-Paul S, P A O, et al. Pembrolizumab in patients with programmed death ligand 1-positive advanced ovarian cancer: analysis of KEYNOTE-028[J]. Gynecol Oncol. 2019;152(2):243-250.

41. Matulonis UA, Shapira-Frommer R, A D S, et al. Antitumor activity and safety of pembrolizumab in patients with advanced recurrent ovarian cancer: results from the phase II KEYNOTE-100 study[J]. Ann Oncol. 2019;30(7):1080-1087.

42. Bamberger ES, Perrett CW. Angiogenesis in epithelian ovarian cancer[J]. Mol Pathol. 2002;55(6):348-359.

43. Gonzalez H, Hagerling C, Werb Z. Roles of the immune system in cancer: from tumor initiation to metastatic progression[J]. Genes Dev. 2018;32(19-20):1267-1284. 
44. Fukumura D, Kloepper J, Amoozgar Z, et al. Enhancing cancer immunotherapy using antiangiogenics: opportunities and challenges[J]. Nat Rev Clin Oncol. 2018;15(5):325-340.
45. Huang Y, Goel S, Duda DG, et al. Vascular normalization as an emerging strategy to enhance cancer immunotherapy[J]. Cancer Res. 2013;73(10):2943-2948.

\section{Publish your work in this journal}

Drug Design, Development and Therapy is an international, peerreviewed open-access journal that spans the spectrum of drug design and development through to clinical applications. Clinical outcomes, patient safety, and programs for the development and effective, safe, and sustained use of medicines are a feature of the journal, which has also been accepted for indexing on PubMed Central. The manuscript management system is completely online and includes a very quick and fair peer-review system, which is all easy to use. Visit http://www. dovepress.com/testimonials.php to read real quotes from published authors.

Submit your manuscript here: https://www.dovepress.com/drug-design-development-and-therapy-journal 